\title{
MONOGENIC CYCLOTOMIC COMPOSITIONS
}

\author{
JOSHUA HARRINGTON AND LENNY JONES
}

\begin{abstract}
Let $m$ and $n$ be positive integers, and let $p$ be a prime. Let $T(x)=\Phi_{p^{m}}\left(\Phi_{2^{n}}(x)\right)$, where $\Phi_{k}(x)$ is the cyclotomic polynomial of index $k$. In this article, we prove that $T(x)$ is irreducible over $\mathbb{Q}$ and that$$
\left\{1, \theta, \theta^{2}, \ldots, \theta^{2^{n-1} p^{m-1}(p-1)-1}\right\}
$$

is a basis for the ring of integers of $\mathbb{Q}(\theta)$, where $T(\theta)=0$.
\end{abstract}

\section{INTRODUCTION}

In this article, unless stated otherwise, polynomials $f(x) \in \mathbb{Z}[x]$ are assumed to be monic, and when we say $f(x)$ is "irreducible", we mean irreducible over $\mathbb{Q}$. Let $K$ be an algebraic number field of degree $n$ over $\mathbb{Q}$. For any $\theta \in K$, we let $\Delta(\theta):=\Delta\left(1, \theta, \theta^{2}, \ldots, \theta^{n-1}\right)$ denote the discriminant of $\theta$. Similarly, we let $\Delta(f)$ and $\Delta(K)$ denote the discriminants over $\mathbb{Q}$, respectively, of the polynomial $f(x)$ and the field $K$. If $f(x)$ is irreducible, with $f(\theta)=0$ and $K=\mathbb{Q}(\theta)$, then we have the well-known equation [5]

$$
\Delta(f)=\Delta(\theta)=\left[\mathbb{Z}_{K}: \mathbb{Z}[\theta]\right]^{2} \Delta(K),
$$

where $\mathbb{Z}_{K}$ is the ring of integers of $K$. We say $f(x)$ is monogenic if $f(x)$ is irreducible and $\left[\mathbb{Z}_{K}: \mathbb{Z}[\theta]\right]=1$, or equivalently from (1.1) that $\Delta(f)=\Delta(K)$. In this situation, $\left\{1, \theta, \theta^{2}, \ldots, \theta^{n-1}\right\}$ is a basis for $\mathbb{Z}_{K}$ referred to as a power basis. The existence of a power basis makes calculations much easier in $\mathbb{Z}_{K}$. A classic example is the cyclotomic field $K=\mathbb{Q}(\zeta)$, where $\zeta$ is a primitive $n$th root of unity 23. In this case, $\mathbb{Z}_{K}=\mathbb{Z}[\zeta]$ and so $\mathbb{Z}_{K}$ has the power basis $\left\{1, \zeta, \ldots, \zeta^{\phi(n)-1}\right\}$, where $\phi(n)$ is Euler's totient function. The minimal polynomial for $\zeta$ over $\mathbb{Q}$ is the cyclotomic polynomial of index $n$, which we denote as $\Phi_{n}(x)$. That is, $\Phi_{n}(x)$ is monogenic of degree $\phi(n)$.

Aside from the cyclotomic polynomials, there are many polynomials that are monogenic. In fact, it is shown in 3 that the density of the irreducible monogenic polynomials of degree $n$ is $6 / \pi^{2} \approx .607927$. However, finding specific infinite families of monogenic polynomials can be challenging. See for example [1,2,4,9, 12, 14, 17, 20, 22 .

Motivated by research concerning power bases of relative extensions 10,11, 15, [16, we are led to ask the following basic related question:

$$
\text { If } f(x) \text { and } g(x) \text { are monogenic, when is } f(g(x)) \text { monogenic? }
$$

It is not shocking that a complete answer to (1.2) should be difficult to achieve. What is somewhat surprising is that the answer to (1.2) requires some effort to

Date: September 10, 2019.

2010 Mathematics Subject Classification. Primary 11R04, Secondary 11R09, 11R32, 12F12.

Key words and phrases. monogenic, irreducible, cyclotomic polynomial, composition. 
unravel, even in seemingly "easy" situations. For example, although $f(x)=x^{2}+17$, $g(x)=x^{2}+5, f(g(x))=x^{4}+10 x^{2}+42$ and $g(f(x))=x^{4}+34 x^{2}+294$ are all irreducible, and both $f(x)$ and $g(x)$ are monogenic, it turns out that $f(g(x))$ is monogenic but $g(f(x))$ is not monogenic. In this article, we focus our investigation on compositions of certain cyclotomic polynomials. More precisely, we prove the following.

Theorem 1.1. Let $m$ and $n$ be positive integers, and let $p$ be a prime. Define

$$
T(x):=\Phi_{p^{m}}\left(\Phi_{2^{n}}(x)\right),
$$

where $\Phi_{k}(x)$ is the cyclotomic polynomial of index $k$. Then $T(x)$ is irreducible and monogenic.

All computer computations in this article were done using either MAGMA, Maple or Sage.

\section{Preliminaries}

We first present some standard terminology and known facts.

Definition 2.1. [5] Let $f(x)$ and $g(x)$ be polynomials over an integral domain $\mathcal{R}$ with respective leading coefficients $a$ and $b$, and respective degrees $m$ and $n$. Let $K$ be the quotient field of $\mathcal{R}$, and let $\bar{K}$ be an algebraic closure of $K$. Suppose that the roots of $f(x)$ and $g(x)$ in $\bar{K}$ are, respectively, $r_{1}, r_{2}, \ldots, r_{m}$ and $s_{1}, s_{2}, \ldots, s_{n}$. Then the resultant of $f(x)$ and $g(x)$, denoted $R(f, g)$, is defined as

$$
R(f, g):=a^{n} b^{m} \prod_{\substack{1 \leq i \leq m \\ 1 \leq j \leq n}}\left(r_{i}-s_{j}\right) .
$$

The discriminant of $f(x)$, denoted $\Delta(f)$ is defined as

$$
\Delta(f):=\frac{(-1)^{m(m-1) / 2}}{a} R\left(f, f^{\prime}\right)=(-1)^{m(m-1) / 2} a^{2 m-2} \prod_{i \neq j}\left(r_{i}-r_{j}\right) .
$$

Proposition 2.2. [5] Let $f(x)$ and $g(x)$ be polynomials as in Definition 2.1.

(1) $R(f, g)=(-1)^{m n} R(g, f)$,

(2) $R(f, g \cdot h)=R(f, g) R(f, h)$, where $h(x)$ is any polynomial over $\mathcal{R}$,

(3) $R(f, g)=a^{n} \prod_{1 \leq i \leq m} g\left(r_{i}\right)$,

(4) Let $c \in \mathcal{R}$. Let $u(x)=f(x+c)$ and $v(x)=g(x+c)$. Then $R(u, v)=R(f, g)$.

(5) $f(x)$ and $g(x)$ have a root in common in $\bar{K}$ if and only if $R(f, g)=0$.

Definition 2.3. Let $p$ be a prime and let

$$
f(x)=a_{n} x^{n}+a_{n-1} x^{n-1}+\cdots+a_{1} x+a_{0} \in \mathbb{Z}[x] .
$$

We say $f(x)$ is $p$-Eisenstein if

$a_{n} \not \equiv 0 \quad(\bmod p), \quad a_{0} \equiv a_{1} \equiv \cdots \equiv a_{n-1} \equiv 0 \quad(\bmod p) \quad$ and $\quad a_{0} \not \equiv 0 \quad\left(\bmod p^{2}\right)$.

Theorem 2.4. [19] (Eisenstien's Irreducibility Criterion) Let $p$ be a prime and let $f(x) \in \mathbb{Z}[x]$ be $p$-Eisenstein. Then $f(x)$ is irreducible.

Theorem 2.5. Let $n$ be a positive integer, and let $\Phi_{n}(x)$ denote the cyclotomic polynomial of index $n$. 
(1) 21] Let $p$ be a prime. Then

$$
\Phi_{n}\left(x^{p}\right)=\left\{\begin{array}{ccc}
\Phi_{p n}(x) & \text { if } n \equiv 0 & (\bmod p) \\
\Phi_{n}(x) \Phi_{p n}(x) & \text { if } n \neq 0 & (\bmod p)
\end{array}\right.
$$

(2) [23] Let $p$ be a prime, and let $m$ be a positive integer. Then

$$
\begin{gathered}
\Delta\left(\Phi_{p^{m}}(x)\right)=\varepsilon p^{p^{m-1}(p m-m-1)}, \\
\text { where } \varepsilon=\left\{\begin{array}{rr}
-1 & \text { if } p^{m}=4 \text { or } p \equiv 3 \quad \text { otherwise. }
\end{array} \quad(\bmod 4)\right.
\end{gathered}
$$

(3) [18] Let $q$ be a prime such that $q \nmid n$. Let $\operatorname{ord}_{n}(q)$ denote the order of $q$ modulo $n$. Then $\Phi_{n}(x)$ factors modulo $q$ into a product of $\phi(n) / \operatorname{ord}_{n}(q)$ distinct irreducible polynomials, each of degree $\operatorname{ord}_{n}(q)$. Additionally, for any positive integer $m$,

$$
\Phi_{q^{m} n}(x) \equiv \Phi_{n}(x)^{\phi\left(q^{m}\right)} \quad(\bmod q)
$$

(4) [8] Let $m$ and $n$ be integers such that $0<m<n$. Then

$$
R\left(\Phi_{m}, \Phi_{n}\right)=\left\{\begin{array}{cl}
p^{\phi(m)} & \text { if } n / m=p^{a} \text { for some prime } p \text { and integer } a \geq 1 \\
1 & \text { otherwise. }
\end{array}\right.
$$

The next lemma follows by a straightforward induction on $n$.

Lemma 2.6. Let $G(x), H(x) \in \mathbb{Z}[x]$, and let $q$ be a prime. If $G(x) \equiv H(x)$ $(\bmod q)$, then

$$
G(x)^{q^{n}} \equiv H(x)^{q^{n}} \quad\left(\bmod q^{n+1}\right) \quad \text { for all } n \geq 1
$$

The following theorem gives a formula for the discriminant of the composition of two polynomials. To the best of our knowledge, this result is originally due to John Cullinan [7] and does not appear in the literature. Hence, for the sake of completeness, we present a proof here.

Theorem 2.7. Let $f(x)$ and $g(x)$ be polynomials in $\mathbb{Q}[x]$, with respective leading coefficients $a$ and $b$, and respective degrees $m$ and $n$. Then

$$
\Delta(f \circ g)=(-1)^{m^{2} n(n-1) / 2} \cdot a^{n-1} b^{m(m n-n-1)} \Delta(f)^{n} R\left(f \circ g, g^{\prime}\right)
$$


Proof. Note that the degree of $f(g(x))$ is $m n$, the leading coefficient of $f(g(x))$ is $a b^{m}$, the degree of $f^{\prime}(x)$ is $m-1$ and the degree of $f^{\prime}(g(x))$ is $(m-1) n$. Then

$$
\begin{aligned}
\Delta(f \circ g) & =\frac{(-1)^{m n(m n-1) / 2}}{a b^{m}} \cdot R\left(f \circ g,(f \circ g)^{\prime}\right) \\
& =\frac{(-1)^{m n(m n-1) / 2}}{a b^{m}} \cdot R\left(f \circ g,\left(f^{\prime} \circ g\right) \cdot g^{\prime}\right) \\
& =\frac{(-1)^{m n(m n-1) / 2}}{a b^{m}} \cdot R\left(f \circ g, f^{\prime} \circ g\right) \cdot R\left(f \circ g, g^{\prime}\right) \quad(\text { Proposition 2.2) } \\
& =\frac{(-1)^{m n(m n-1) / 2}}{a b^{m}} \cdot\left(\left(a b^{m}\right)^{(m-1) n} \prod_{\{\alpha: f(g(\alpha))=0\}} f^{\prime}(g(\alpha))\right) R\left(f \circ g, g^{\prime}\right) \\
& =\frac{(-1)^{m n(m n-1) / 2}}{a b^{m}} \cdot\left(a b^{m}\right)^{(m-1) n}\left(\prod_{\{\theta: f(\theta)=0\}} f^{\prime}(\theta)\right)^{n} R\left(f \circ g, g^{\prime}\right) \\
& =\frac{(-1)^{m n(m n-1) / 2}}{a b^{m}} \cdot\left(a b^{m}\right)^{(m-1) n}\left(\frac{1}{a^{m-1}} R\left(f, f^{\prime}\right)\right)^{n} R\left(f \circ g, g^{\prime}\right) \\
& =\frac{(-1)^{m n(m n-1) / 2}}{a b^{m}} \cdot \frac{\left(a b^{m}\right)^{(m-1) n}}{a^{(m-1) n}} R\left(f, f^{\prime}\right)^{n} R\left(f \circ g, g^{\prime}\right) \quad(\operatorname{Proposition} 2.2) \\
& =\frac{(-1)^{m n(m n-1) / 2}}{a b^{m}} \cdot \frac{\left(a b^{m}\right)^{(m-1) n}}{a^{(m-1) n}}\left(\frac{a \Delta(f)}{(-1)^{m(m-1) / 2}}\right)^{n} R\left(f \circ g, g^{\prime}\right) \\
& =(-1)^{m^{2} n(n-1) / 2} \cdot a^{n-1} b^{m(m n-n-1)} \Delta(f)^{n} R\left(f \circ g, g^{\prime}\right) .
\end{aligned}
$$

The following special case of Theorem 2.7 will be useful to us.

Corollary 2.8. Let $f(x) \in \mathbb{Q}[x]$, such that a is the leading coefficient of $f(x)$, and $m=\operatorname{deg}(f)$. If $g(x)=b x^{n}+c \in \mathbb{Q}[x]$, then

$$
\Delta(f \circ g)=(-1)^{m n(n-1)(m+2 n) / 2} a^{n-1} b^{m(m n-1)} \Delta(f)^{n} n^{m n} f(c)^{n-1} .
$$

Proof. Note that $\operatorname{deg}(f \circ g)=m n$ and $\operatorname{deg}\left(g^{\prime}\right)=n-1$. Then, using Theorem 2.7 and Proposition 2.2 we have

$$
\begin{aligned}
\Delta(f \circ g) & =(-1)^{m^{2} n(n-1) / 2} a^{n-1} b^{(m-1) n m-m} \Delta(f)^{n} R\left(f \circ g, g^{\prime}\right) \\
& =(-1)^{m^{2} n(n-1) / 2} a^{n-1} b^{(m-1) n m-m} \Delta(f)^{n}(-1)^{m n(n-1)} R\left(g^{\prime}, f \circ g\right) \\
& =(-1)^{m^{2} n(n-1) / 2} a^{n-1} b^{(m-1) n m-m} \Delta(f)^{n}(-1)^{m n(n-1)}(b n)^{m n} \prod_{i=1}^{n-1} f(g(0)) \\
& =(-1)^{m^{2} n(n-1) / 2} a^{n-1} b^{(m-1) n m-m} \Delta(f)^{n}(-1)^{m n(n-1)}(b n)^{m n} f(c)^{n-1} \\
& =(-1)^{m n(n-1)(m+2 n) / 2} a^{n-1} b^{m(m n-1)} \Delta(f)^{n} n^{m n} f(c)^{n-1} .
\end{aligned}
$$


Theorem 2.9. [6] Let $p$ be a prime and let $f(x) \in \mathbb{Z}[x]$ be a monic p-Eisenstien polynomial with $\operatorname{deg}(f)=n$. Let $K=\mathbb{Q}(\theta)$, where $f(\theta)=0$. If $n \not \equiv 0(\bmod p)$, then $p^{n-1} \| \Delta(K)$.

Theorem 2.10 (Dedekind). [5] Let $K=\mathbb{Q}(\theta)$ be a number field, $T(x) \in \mathbb{Z}[x]$ the monic minimal polynomial of $\theta$, and $\mathbb{Z}_{K}$ the ring of integers of $K$. Let $p$ be a prime number and let $*$ denote reduction of $*$ modulo $p$ (in $\mathbb{Z}, \mathbb{Z}[x]$ or $\mathbb{Z}[\theta]$ ). Let

$$
\bar{T}(x)=\prod_{i=1}^{k} \overline{t_{i}}(x)^{e_{i}}
$$

be the factorization of $T(x)$ modulo $p$ in $\mathbb{F}_{p}[x]$, and set

$$
g(x)=\prod_{i=1}^{k} t_{i}(x),
$$

where the $t_{i}(x) \in \mathbb{Z}[x]$ are arbitrary monic lifts of the $\overline{t_{i}}(x)$. Let $h(x) \in \mathbb{Z}[x]$ be a monic lift of $\bar{T}(x) / \bar{g}(x)$ and set

$$
F(x)=\frac{g(x) h(x)-T(x)}{p} \in \mathbb{Z}[x] .
$$

Then

$$
\left[\mathbb{Z}_{K}: \mathbb{Z}[\theta]\right] \not \equiv 0 \quad(\bmod p) \Longleftrightarrow \operatorname{gcd}(\bar{F}, \bar{g}, \bar{h})=1 \text { in } \mathbb{F}_{p}[x]
$$

\subsection{The Proof of Theorem 1.1.}

Proof. Note that when $n=1$, the theorem is trivially true since in that case $T(x)$ is simply a translation of $\Phi_{p^{m}}(x)$. So, assume that $n \geq 2$. We first show that $T(x)$ is irreducible by showing that $T(x)$ is $p$-Eisenstein. Since $\Phi_{1}(x)=x-1$ and $\Phi_{2^{n}}(x)=x^{2^{n-1}}+1$, we have from part (3) of Theorem 2.5 with $q=p$ and $n=1$ that

$$
T(x)=\Phi_{p^{m}}\left(\Phi_{2^{n}}(x)\right) \equiv \Phi_{1}\left(\Phi_{2^{n}}(x)\right)^{p^{m-1}(p-1)} \equiv x^{2^{n-1} p^{m-1}(p-1)} \quad(\bmod p) .
$$

It follows that only the leading coefficient of $T(x)$ is not divisible by $p$. Since the constant term of $T(x)$ is $T(0)=\Phi_{p^{m}}\left(\Phi_{2^{n}}(0)\right)=\Phi_{p^{m}}(1)=p$, we conclude that $T(x)$ is $p$-Eisenstein.

Next, using Corollary 2.8 and part (2) of Theorem 2.5. we have that

$$
\begin{aligned}
\Delta(T) & =\left(\varepsilon p^{p^{m-1}(p m-m-1)}\right)^{2^{n-1}}\left(2^{n-1}\right)^{p^{m-1}(p-1) 2^{n-1}} \Phi_{p^{m}}(1)^{2^{n-1}-1} \\
& =\varepsilon^{2^{n-1}} 2^{(n-1) 2^{n-1} p^{m-1}(p-1)} p^{m 2^{n-1} p^{m-1}(p-1)-1} .
\end{aligned}
$$

Suppose that $T(\theta)=0$, and let $K=\mathbb{Q}(\theta)$. We now use Theorem 2.10 to show that neither 2 nor $p$ divides $\left[\mathbb{Z}_{K}: \mathbb{Z}[\theta]\right]$.

We address the prime $p$ first. From (2.1), we can let $g(x)=x$ and $g(x) h(x)=$ $x^{2^{n-1} p^{m-1}(p-1)}$ in Theorem 2.10, so that

$$
F(x)=\frac{x^{2^{n-1} p^{m-1}(p-1)}-T(x)}{p} .
$$

It follows that $\bar{F}(0)=-1$. Consequently, $\operatorname{gcd}(\bar{F}, \bar{g})=1$ in $\mathbb{F}_{p}[x]$, and $\left[\mathbb{Z}_{K}: \mathbb{Z}[\theta]\right] \not \equiv$ $0(\bmod p)$ by Theorem 2.10 . 
If $p=2$, there is nothing else to do. So, assume that $p \neq 2$. To address the prime 2 , first note that

$$
T(x) \equiv \Phi_{p^{m}}\left((x+1)^{2^{n-1}}\right) \equiv\left(\Phi_{p^{m}}(x+1)\right)^{2^{n-1}} \quad(\bmod 2) .
$$

Let $a=\phi\left(p^{m}\right)$ and $b=\operatorname{ord}_{p^{m}}(2)$. By part (3) of Theorem 2.5 with $n=p^{m}$ and $q=2$, we conclude that

$$
\Phi_{p^{m}}(x+1) \equiv \prod_{i=1}^{a / b} t_{i}(x) \quad(\bmod 2),
$$

where the $t_{i}(x)$ are distinct irreducible polynomials each of degree $b$. Hence, we may take

$$
g(x)=\prod_{i=1}^{a / b} t_{i}(x) \quad \text { and } \quad g(x) h(x)=\left(\prod_{i=1}^{a / b} t_{i}(x)\right)^{2^{n-1}}
$$

in Theorem 2.10, Then, from (2.3), we can write

$$
g(x)=\Phi_{p^{m}}(x+1)+2 r(x)=\frac{(x+1)^{p^{m}}-1}{(x+1)^{p^{m-1}}-1}+2 r(x),
$$

for some polynomial $r(x) \in \mathbb{Z}[x]$. For $n \geq 2$, define

$$
F_{n}(x):=\frac{g(x)^{2^{n-1}}-\Phi_{p^{m}}\left(x^{2^{n-1}}+1\right)}{2} \in \mathbb{Z}[x] .
$$

By Lemma 2.6. we deduce that $g(x)^{2^{n}} \equiv g\left(x^{2}\right)^{2^{n-1}}(\bmod 4)$ for $n \geq 2$ so that

$$
g(x)^{2^{n}}-\Phi_{p^{m}}\left(x^{2^{n}}+1\right) \equiv g\left(x^{2}\right)^{2^{n-1}}-\Phi_{p^{m}}\left(\left(x^{2}\right)^{2^{n-1}}+1\right) \quad(\bmod 4) .
$$

That is,

$$
2 F_{n+1}(x) \equiv 2 F_{n}\left(x^{2}\right) \quad(\bmod 4) \quad \text { for } n \geq 2 .
$$

Hence, for all $n \geq 2$, it follows that

$$
F_{n+1}(x) \equiv F_{n}\left(x^{2}\right) \equiv F_{n}(x)^{2} \quad(\bmod 2) .
$$

Consequently, to show that $\operatorname{gcd}\left(\overline{F_{n}}, \bar{g}\right)=1$ for all $n \geq 2$, it is enough to show that $\operatorname{gcd}\left(\overline{F_{2}}, \bar{g}\right)=1$. Let

$$
\begin{gathered}
A(x)=(x+1)^{p^{m}}-1, \quad B(x)=\left(x^{2}+1\right)^{p^{m-1}}-1, \\
C(x)=\left(x^{2}+1\right)^{p^{m}}-1 \quad \text { and } \quad D(x)=(x+1)^{p^{m-1}}-1 .
\end{gathered}
$$

Using (2.5) and (2.6), we have that

$$
\begin{aligned}
F_{2}(x) & =\frac{\left(\frac{A(x)}{D(x)}+2 r(x)\right)^{2}-\frac{C(x)}{B(x)}}{2} \\
& =\frac{A(x)^{2} B(x)-C(x) D(x)^{2}}{2 B(x) D(x)^{2}}+2\left(\frac{r(x) A(x)+r(x)^{2} D(x)}{D(x)}\right) .
\end{aligned}
$$


Expanding $A(x)^{2}$, we get

$$
\begin{aligned}
A(x)^{2} & =(x+1)^{2 p^{m}}-2(x+1)^{p^{m}}+1 \\
& =\left(x^{2}+1+2 x\right)^{p^{m}}-2(x+1)^{p^{m}}+1 \\
& =\left(\left(x^{2}+1\right)^{p^{m}}+p^{m}\left(x^{2}+1\right)^{p^{m}-1}(2 x)+\cdots+(2 x)^{p^{m}}\right)-2(x+1)^{p^{m}}+1 \\
& =\left(x^{2}+1\right)^{p^{m}}+2 p^{m} x\left(x^{2}+1\right)^{p^{m}-1}+4 u(x)-2(x+1)^{p^{m}}+1,
\end{aligned}
$$

where

$$
4 u(x)=\left(\begin{array}{c}
p^{m} \\
2
\end{array}\right)\left(x^{2}+1\right)^{p^{m}-2}(2 x)^{2}+\cdots+\left(\begin{array}{c}
p^{m} \\
p^{m}-1
\end{array}\right)\left(x^{2}+1\right)(2 x)^{p^{m}-1}+(2 x)^{p^{m}} .
$$

Similarly,

$$
D(x)^{2}=\left(x^{2}+1\right)^{p^{m-1}}+2 p^{m-1} x\left(x^{2}+1\right)^{p^{m-1}-1}+4 v(x)-2(x+1)^{p^{m-1}}+1,
$$

where

$$
4 v(x)=\left(\begin{array}{c}
p^{m-1} \\
2
\end{array}\right)\left(x^{2}+1\right)^{p^{m-1}-2}(2 x)^{2}+\cdots+(2 x)^{p^{m-1}} .
$$

Substituting the expressions above for $A(x)^{2}$ and $D(x)^{2}$ into the numerator of the first term of (2.7) and cancelling the factor of 2 yields

$$
F_{2}(x)=\frac{E(x)}{B(x) D(x)^{2}}+2\left(\frac{u(x) B(x)-v(x) C(x)}{B(x) D(x)^{2}}+\frac{r(x) A(x)+r(x)^{2} D(x)}{D(x)}\right),
$$

where

$$
\begin{aligned}
& E(x)=-\left(x^{2}+1\right)^{p^{m}}+\left(x^{2}+1\right)^{p^{m-1}}+p^{m} x\left(x^{2}+1\right)^{p^{m}-1+p^{m-1}} \\
&-p^{m} x\left(x^{2}+1\right)^{p^{m}-1}-(x+1)^{p^{m}}\left(x^{2}+1\right)^{p^{m-1}} \\
&+(x+1)^{p^{m}}-\left(x^{2}+1\right)^{p^{m}-1+p^{m-1}} p^{m-1} x \\
&+\left(x^{2}+1\right)^{p^{m}}(x+1)^{p^{m-1}} \\
&+p^{m-1} x\left(x^{2}+1\right)^{p^{m-1}-1}-(x+1)^{p^{m-1}} .
\end{aligned}
$$

Then, reduction of $F_{2}(x)$ modulo 2 produces

$$
\overline{F_{2}}(x)=\frac{\bar{E}(x)}{\left((x+1)^{p^{m-1}}-1\right)^{4}},
$$

where

$$
\begin{aligned}
\bar{E}(x) & =(x+1)^{2 p^{m}}+(x+1)^{2 p^{m-1}}+x(x+1)^{2\left(p^{m}-1\right)}+(x+1)^{p^{m}}(x+1)^{2 p^{m-1}} \\
+ & (x+1)^{p^{m}}+(x+1)^{2 p^{m}}(x+1)^{p^{m-1}}+x(x+1)^{2\left(p^{m-1}-1\right)}+(x+1)^{p^{m-1}} .
\end{aligned}
$$

We claim that $\operatorname{gcd}\left(\overline{F_{2}}, \bar{g}\right)=1$. To establish this claim, assume to the contrary that there exists $\alpha$ in some algebraic closure of $\mathbb{F}_{2}$ such that

$$
\bar{g}(\alpha)=0=\overline{F_{2}}(\alpha) .
$$


Then, from (2.3) and (2.4), we have $\Phi_{p^{m}}(\alpha+1)=0$ so that $(\alpha+1)^{p^{m}}=1$. Hence, it follows from (2.8) that

$$
\begin{aligned}
\overline{F_{2}}(\alpha) & =\frac{\alpha(\alpha+1)^{2\left(p^{m-1}-1\right)}\left(\left((\alpha+1)^{p^{m-1}}\right)^{(p-1) / 2}-1\right)^{4}}{\left((\alpha+1)^{p^{m-1}}-1\right)^{4}} \\
& =\alpha(\alpha+1)^{2\left(p^{m-1}-1\right)}\left(\prod_{\substack{d \mid \frac{p-1}{2} \\
d>1}} \Phi_{d}\left((\alpha+1)^{p^{m-1}}\right)\right)^{4} .
\end{aligned}
$$

Thus, since $\overline{F_{2}}(\alpha)=0$ and both $\bar{g}(0)$ and $\bar{g}(-1)$ are nonzero, we conclude that $\Phi_{p^{m}}(x+1)$ and $\Phi_{d}\left((x+1)^{p^{m-1}}\right)$ have the root $\alpha$ in common for some divisor $d>1$ of $(p-1) / 2$. Repeated application of part (11) of Theorem 2.5 yields

$$
\Phi_{d}\left((x+1)^{p^{m-1}}\right)=\prod_{j=0}^{m-1} \Phi_{p^{j} d}(x+1) .
$$

Therefore, $\Phi_{p^{m}}(x+1)$ and $\Phi_{p^{j} d}(x+1)$ have the root $\alpha$ in common for some $j$ with $0 \leq j \leq m-1$. However, recall that $d$ is a divisor of $(p-1) / 2$ with $d>1$ and note that $p^{j} d<p^{m}$. Hence, $\operatorname{gcd}(d, p)=1$ and $p^{m} / p^{j} d$ is not an integer. Thus, from part (4) of Theorem 2.5, we have that $R\left(\Phi_{p^{j}}, \Phi_{p^{m}}\right)=1$, which contradicts (2.9) by part (5) of Proposition 2.2. Therefore, by Theorem 2.10 we have established that $\left[\mathbb{Z}_{K}: \mathbb{Z}[\theta]\right] \not \equiv 0(\bmod 2)$, which completes the proof of the theorem.

\section{Final Remarks}

We certainly do not claim that Theorem 1.1 represents the most general result possible, since computationally there appear to be many other situations where the composition of two cyclotomic polynomials is indeed monogenic. The main difficulty in establishing a result that is more general than Theorem 1.1 arises from the handling of the prime divisors of the resultant appearing in the formula given in Theorem 2.7 for the discriminant of the composition. On the other hand, while many monogenic cyclotomic compositions exist that are not described by Theorem 1.1. we should point out that slight deviations from the polynomials in Theorem 1.1 yield compositions that are not monogenic. For example, it is not difficult to show that $\Phi_{4}\left(\Phi_{3^{n}}(x)\right)$ and $\Phi_{3}\left(\Phi_{5^{n}}(x)\right)$ are reducible for all $n \geq 1$, and although $\Phi_{2}\left(\Phi_{25}(x)\right)$ is irreducible, it is not monogenic since the ring of integers does not possess a power basis. Perhaps these exceptions can be completely determined, but that is a topic for future research.

\section{REFERENCES}

[1] S. Ahmad, T. Nakahara and A. Hameed, On certain pure sextic fields related to a problem of Hasse, Internat. J. Algebra Comput. 26 (2016), no. 3, 577-583.

[2] S. Ahmad, T. Nakahara and S. M. Husnine, Power integral bases for certain pure sextic fields, Int. J. Number Theory 10 (2014), no. 8, 2257-2265. 
[3] M. Bhargava, A. Shankar and X. Wang, Squarefree values of polynomial discriminants I, https://arxiv.org/abs/1611.09806

[4] D. W. Boyd, G. Martin and M. Thom, Squarefree values of trinomial discriminants, LMS J. Comput. Math. 18 (2015), no. 1, 148-169.

[5] H. Cohen, A Course in Computational Algebraic Number Theory, SpringerVerlag, 2000.

[6] K. Conrad, www.math.uconn.edu/ kconrad/blurbs/gradnumthy/totram.pdf

[7] https://studylib.net/doc/8187082/the-discriminant-of-a-composition-of-two

[8] G. Dresden, Resultants of cyclotomic polynomials, Rocky Mountain J. Math. 42 (2012), no. 5, 1461-1469.

[9] D. Eloff, B. Spearman and K. Williams, $A_{4}$-sextic fields with a power basis, Missouri Journal of Mathematical Sciences 19 (2007), 188-194.

[10] I. Gaál and M. Pohst, Computing power integral bases in quartic relative extensions, J. Number Theory 85 (2000), no. 2, 201-219.

[11] I. Gaál, Power integral bases in cubic relative extensions, Experiment. Math. 10 (2001), no. 1, 133-139.

[12] I. Gaál, Diophantine equations and power integral bases. New computational methods., Birkhuser Boston, Inc., Boston, MA, (2002).

[13] I. Gaál and L. Remete, Power integral bases in a family of sextic fields with quadratic subfields, Tatra Mt. Math. Publ. 64 (2015), 59-66.

[14] I. Gaál and L. Remete, Integral bases and monogenity of pure fields, J. Number Theory 173 (2017), 129-146.

[15] I. Gaál, L. Remete and T. Szabó, Calculating power integral bases by using relative power integral bases, Funct. Approx. Comment. Math. 54 (2016), no. $2,141-149$.

[16] I. Gaál and T. Szabó, Relative power integral bases in infinite families of quartic extensions of quadratic fields, JP J. Algebra Number Theory Appl. 29 (2013), no. 1, 31-43.

[17] T. A. Gassert, Discriminants of Chebyshev radical extensions, J. Théor. Nombres Bordeaux 26 (2014), no. 3, 607-634.

[18] W. J. Guerrier, The factorization of the cyclotomic polynomials mod $p$, Amer. Math. Monthly 75 (1968) 46. 
[19] Kenneth Ireland and Michael Rosen, A Classical Introduction to Modern Number Theory, Second Edition, Graduate Texts in Mathematics, 84, SpringerVerlag, New York, 1990.

[20] B. Jhorar and S. Khanduja, On power basis of a class of algebraic number fields, Int. J. Number Theory 12 (2016), no. 8, 2317-2321.

[21] T. Nagell, Introduction to Number Theory, New York: Wiley, 1951. Arch. Math. (Basel) 102 (2014), no. 6, 545-554.

[22] B. K. Spearman, Monogenic $A_{4}$ quartic fields, International Mathematical Forum, 1, (2006), no. 40, 1969-1974.

[23] L. C. Washington, Introduction to cyclotomic fields, Second edition, Graduate Texts in Mathematics, 83, Springer-Verlag, New York, 1997.

Department of Mathematics, Cedar Crest College, Allentown, Pennsylvania, Usa

E-mail address, Joshua Harrington: Joshua.Harrington@cedarcrest.edu

Professor Emeritus, Department of Mathematics, Shippensburg University, ShippensBurg, Pennsylvania 17257, USA

E-mail address, Lenny Jones: lkjone@ship.edu 